\title{
Fiber-optic interferometric two-dimensional scattering-measurement system
}

\author{
Yizheng Zhu, Michael G. Giacomelli, and Adam Wax* \\ Department of Biomedical Engineering, Duke University, Durham, North Carolina 27708, USA \\ *Corresponding author: a.wax@duke.edu
}

Received February 19, 2010; accepted March 28, 2010;

posted April 16, 2010 (Doc. ID 124362); published May 6, 2010

\begin{abstract}
We present a fiber-optic interferometric system for measuring depth-resolved scattering in two angular dimensions using Fourier-domain low-coherence interferometry. The system is a unique hybrid of the Michelson and Sagnac interferometer topologies. The collection arm of the interferometer is scanned in two dimensions to detect angular scattering from the sample, which can then be analyzed to determine the structure of the scatterers. A key feature of the system is the full control of polarization of both the illumination and the collection fields, allowing for polarization-sensitive detection, which is essential for twodimensional angular measurements. System performance is demonstrated using a double-layer microsphere phantom. Experimental data from samples with different sizes and acquired with different polarizations show excellent agreement with Mie theory, producing structural measurements with subwavelength accuracy. (C) 2010 Optical Society of America

OCIS codes: $120.5820,290.5855,290.4020,120.3180$.
\end{abstract}

Light-scattering techniques, based on analysis of singly scattered (light-scattering spectroscopy) and/or multiply scattered (elastic-scattering spectroscopy) photons, have been widely used to obtain quantitative morphological information of biological scatterers, such as tissues, cells, and subcellular components [1-3]. The structure of a scattering medium can be identified by analysis of the spectroscopic or angular dependence of scattered light, sometimes in combination with polarization dependence $[4,5]$. Various approaches have been successfully exploited to study cell biology and bulk-tissue properties [1-5], but most are noninterferometric in nature and do not provide depth resolution of the scattering medium.

Angle-resolved low-coherence interferometry (a/LCI) adopts an interferometric approach to light scattering. It measures the angular distribution of singly scattered photons to determine the depthresolved structure of a scattering sample by comparing measured scattering distributions with a database of theoretical predictions [6]. A fiber-bundlebased endoscopic a/LCI probe has recently been demonstrated for in vivo measurement of average nuclear diameters, as a means to detect dysplasia in the upper gastrointestinal tract [7]. Aiming to improve the design of this instrument, specifically, to overcome a limitation on the length of the fiber bundle imposed by the manufacturing process, a Mach-Zehnder interferometer-based (MZI) scanningfiber system was recently proposed [8]. These previous a/LCI systems collect angular scattering in only one dimension (1D), owing to instrumental limitations such as the inability to adequately control the polarization of the collected scattering. Measuring scattering in two angular dimensions (2D) is advantageous over 1D measurement in that the additional information can help improve fitting accuracy and may provide access to additional morphological characteristics, such as aspect ratio and orientation of the scatterer.
To address this issue, we present here the next generation of a/LCI, termed the fiber-optic interferometric 2D scattering (FITS) system. The FITS system is based on a novel fiber-optic Michelson-Sagnac hybrid interferometer (MSI) and is superior to the MZI scheme [8] in that it allows for accurate polarization control and the investigation of $2 \mathrm{D}$ polarization-sensitive angular scattering. In this Letter, we introduce the principle behind the MSI approach and then demonstrate the performance of the FITS system with scattering measurements from a two-layer microsphere phantom.

Figure 1(a) shows the FITS system schematic. Light from a Ti:sapphire laser (Coherent, Inc.: $825 \mathrm{~nm}, \Delta \lambda=17 \mathrm{~nm}, 30 \mathrm{~mW}$ ) is coupled into a singlemode-fiber coupler (coupling ratio $\alpha=0.01 \%$ ). The two sensing arms are cleaved, and their facets are placed in the focal plane of a graded index (GRIN) lens

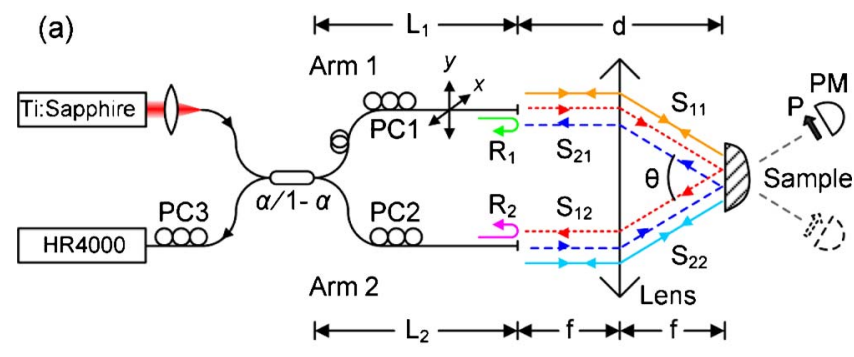

(b)

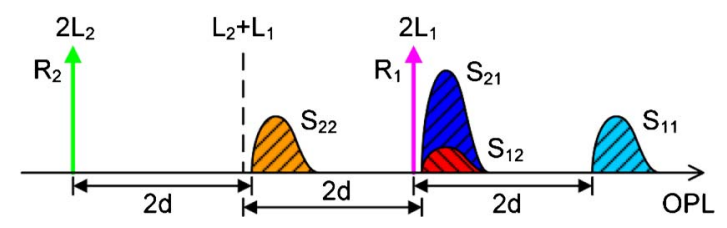

Fig. 1. (Color online) Principle of MSI. (a) Schematic. $d$, optical path length from the fiber ends to the sample; $\theta$, scattering angle; $f$, focal length; PC1-3, polarization controllers; P, polarizer; PM, powermeter; $x$ and $y$, scan directions. (b) Optical path length of the signals. 
(Newport Corp.: 0.23 pitch, $1.8 \mathrm{~mm}$ diameter, $4.4 \mathrm{~mm}$ length) for illumination and collection. The GRIN lens is angled at $8^{\circ}$ on the sample side to avoid specular reflection. The majority of source power is coupled into Arm 2, which serves as the illumination fiber. Its output is collimated via the GRIN lens, illuminating the area of interest on the sample. Arm 1 is the lowpower arm and serves as the collection fiber that receives the light scattered at angle $\theta$. To maximize the detectable angular range, Arm 2 is positioned toward the edge of the GRIN lens, whereas Arm 1 rasterscans in $2 \mathrm{D}$ using a pair of motorized actuators. The polarization of the illumination and collection fields can be tuned independently using polarization controllers (PC1\&2) to be linearly polarized along any direction with an extinction ratio greater than $20 \mathrm{~dB}$, making it possible to measure scattering under any combination of illumination and collection polarization. Signals are detected by a miniature spectrometer (Ocean Optics, Inc.: HR4000).

The MSI scheme resembles a Michelson or Sagnac interferometer, but its operation relies on coherently mixing both the Michelson and Sagnac components, hence the hybrid mode. Three pairs of signals are generated: (1) Michelson signals $R_{1}$ and $R_{2}$, which are the fiber-end reflections and serve as reference signals; (2) Sagnac signals $\mathrm{S}_{12}$ and $\mathrm{S}_{21}$, which are the angular scattering signals and are the signals of interest; and (3) backscattering signals $\mathrm{S}_{11}$ and $\mathrm{S}_{22}$, which can also be considered Michelson signals, but are irrelevant since they only represent scattering in the backscattering direction $\left(180^{\circ}\right)$. For interferometric operation, the MSI matches the optical pathlength (OPL) of $R_{1}$ and $S_{21}$. This is achieved by setting the OPL of the collection arm $L_{1}$ to be longer than that of the illumination arm $L_{2}$ by a specific amount to satisfy a matching condition. Other scattering signals will not be detected, owing to pathlength mismatch and/or attenuated intensity caused by the unbalanced power splitting.

We first discuss the pathlength matching condition. Table 1 lists the OPL of each signal, and Fig. 1(b) illustrates their relative locations assuming $L_{1}$ $>L_{2}$. The OPLs are solely determined by $L_{1}, L_{2}$, and the lens-specific parameter $d$. As a result $R_{1}$ and $S_{21}$ can be pathlength matched by tuning the differential of the two arms, $L_{1}-L_{2}$. Specifically, $\mathrm{S}_{21}\left(\mathrm{~S}_{12}\right)$ can be placed right next to $R_{1}$ in the OPL domain to generate interference, as shown in Fig. 1(b). Hence depthresolved information of $S_{21}\left(S_{12}\right)$ can be acquired using $R_{1}$ as the reference. The matching condition for a hybrid operation can then be written as

$$
L_{1}-L_{2}=2 d \quad \text { or } \quad l_{1}-l_{2}=2 d / n,
$$

where $n$ is the refractive index of the fiber and $l_{1}$ and $l_{2}$ are the physical length of the two arms. Signals $\mathrm{R}_{2}$,
$\mathrm{S}_{11}$, and $\mathrm{S}_{22}$ will not generate detectable interference in this setup, since they are far separated $(\Delta \mathrm{OPL}$ $\sim 2 d)$ from $R_{1}$ and $S_{21}\left(S_{12}\right)$. This is due to a sufficiently large $d(\sim 7.3 \mathrm{~mm}$ for the GRIN lens) that places these high-frequency interference signals beyond the spectrometer's detection range $(\sim 4 \mathrm{~mm})$.

Next we show that a low splitting ratio further attenuates undesirable scattering signals. The primary purpose of the low splitting ratio $(\alpha=0.01 \%)$ is to attenuate the reference signal to achieve proper power balance with the scattering signal. The value of $0.01 \%$ is determined using intensity data from the MZI-based system [8]. Table 1 lists simplified signal intensities, assuming $\alpha \ll 1$ and the scattering coefficients and fiber-end reflectivities (all in lower case) satisfy $s_{11}, s_{22}, s_{12}, s_{21} \ll r_{1}, r_{2} \ll 1$, which are reasonable assumptions for cleaved fibers $(\sim 3.4 \%)$ and scattering of biological samples with small refractive index changes. These intensities show that a small $\alpha$ not only attenuates reference signals but also effectively eliminate irrelevant signals $\mathrm{S}_{11}$ and $\mathrm{S}_{22}$ by a factor of $\alpha$, as well as the overlapping signal, $\mathrm{S}_{12}$, by a factor of $\alpha^{2}$. It is worth noting that the use of a small $\alpha$ is not a requirement for MSI operation, since pathlength matching by itself is sufficient to prevent detection of unwanted signals. A small $\alpha$, however, further isolates the signals of interest by leaving only three signals with substantial strength: sample signal $S_{21}$, reference signal $R_{1}$, and noninterferometric background signal $R_{2}$, which can be frustrated by angle polishing when necessary.

The symmetry of this system points to the fact that $R_{2}$ can also serve as the reference signal, provided that $L_{2}>L_{1}$. The difference is that using the lowpower arm signal $R_{1}$ as the reference offers superior polarization performance to the use of $R_{2}$. If $R_{2}$ were used for reference, the signals $S_{21}$ and $R_{2}$ would travel separate paths through Arms 1 and 2 before being mixed inside the coupler. The distinct paths would make it impossible to determine or control their polarization states inside the coupler owing to disturbance to the fibers. Consequently, there would be no precise control as to which polarization component of the scattering is detected. In the preferred case, where $R_{1}$ provides reference, its mixing with $S_{21}$ occurs at the end of Arm 1, prior to propagation through the fiber. Thus these two signals travel together and fiber disturbance has no effect. The detected polarization component of $\mathrm{S}_{21}$ is determined by the direction of the linearly polarized $R_{1}$ as it exits Arm 1, a parameter that can be measured and adjusted using a polarizer and a power meter. Similarly, the illumination polarization can be adjusted as well. In summary, the MSI scheme allows both the illumination and the collection to be either $p$ or $s$ polarized,

Table 1. OPL and Intensity of MSI Signals

\begin{tabular}{ccccccc}
\hline Signal & $\mathrm{R}_{1}$ & $\mathrm{R}_{2}$ & $\mathrm{~S}_{21}$ & $\mathrm{~S}_{12}$ & $\mathrm{~S}_{11}$ & $\mathrm{~S}_{22}$ \\
\hline OPL & $2 L_{1}$ & $2 L_{2}$ & $L_{1}+L_{2}+2 d$ & $L_{1}+L_{2}+2 d$ & $2 L_{1}+2 d$ & $2 L_{2}+2 d$ \\
Intensity $^{a}$ & $\alpha r_{1}$ & $\alpha r_{2}$ & $s_{21}$ & $\alpha^{2} s_{12}$ & $\alpha s_{11}$ & $\alpha s_{22}$ \\
\hline
\end{tabular}

${ }^{a}$ Normalized to source intensity. 
offering full polarization control and hence 2D capability.

We demonstrate the capabilities of the MSI-based FITS system by collecting 2D angular scattering from a double-layer silicone phantom with embedded microsphere-size standards (Thermo Fisher Scientific, Inc.) with mean diameters of $5.990 \pm 0.045 \mu \mathrm{m}$ and $10.00 \pm 0.05 \mu \mathrm{m}$ and standard deviations of $1.2 \%$ and $0.9 \%$, respectively.

Arm 1 raster scans an area of $1.0 \times 1.8 \mathrm{~mm}^{2}$ $(y \times x)$ with a continuous scan $(0.35 \mathrm{~mm} / \mathrm{s})$ in $x$ and a step scan $(10 \mu \mathrm{m} / \mathrm{step})$ in $y$. A complete scan currently takes $12 \mathrm{~min}$ and generates a $2 \mathrm{D}$ angularscattering distribution containing $90 \times 170$ useful data points, with an angular resolution of $0.212 \%$ step in both directions. At each point of the $2 \mathrm{D}$ distribution, the interference spectrum is Fourier transformed into depth-resolved scattering intensity with a depth resolution of $17.7 \mu \mathrm{m}$. To demonstrate polarization-sensitive measurements, we use $p$-polarized illumination and collect both the $p$ and $s$ components of the scattered field.

Figures 2(a) and 2(b) show the depth-resolved 2D angular scattering of the phantom, where the doublelayer structure is clearly identified. Figures 2(c)-2(j) show that the measured distribution for each layer and each polarization are in good agreement with the predictions of Mie theory. The high-frequency fringes seen in the experimental data are likely due to coherent scattering from adjacent microspheres in the phantom. Such information can be potentially useful for estimation of particle density and spacing.

Figure 3 shows fitting examples along lines A, B, and $\mathrm{C}$ in Fig. 2, producing subwavelength sizing accuracy. Data from lines A and B give scattering distributions that could be measured with the traditional $1 \mathrm{D}$ a/LCI method. The fitting results for line $\mathrm{C}$ demonstrates that accurate sizing can also be ob-
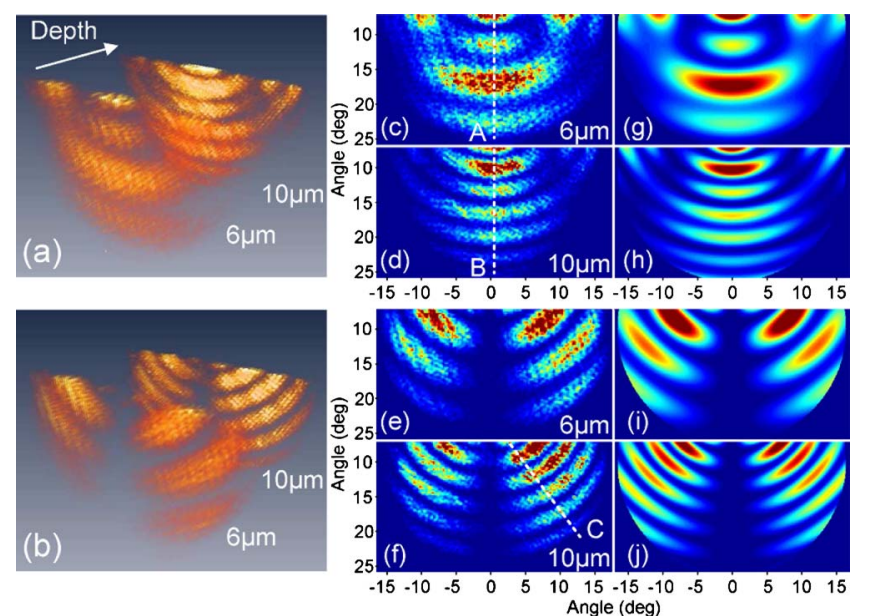

Fig. 2. (Color online) Scattering of the double-layer phantom under $p$-polarized illumination. (a), (b) Depth-resolved 2D angular scattering for $p$ - (Media 1) and $s$-polarized (Media 2) scattering. (c), (d) $p$ - and (e), (f) $s$-polarized 2D scattering for 6 and $10 \mu \mathrm{m}$ layers, respectively. (g), (h) and (i), (j) Corresponding Mie theory simulations. A, B, and C are lines along which data fitting is executed.
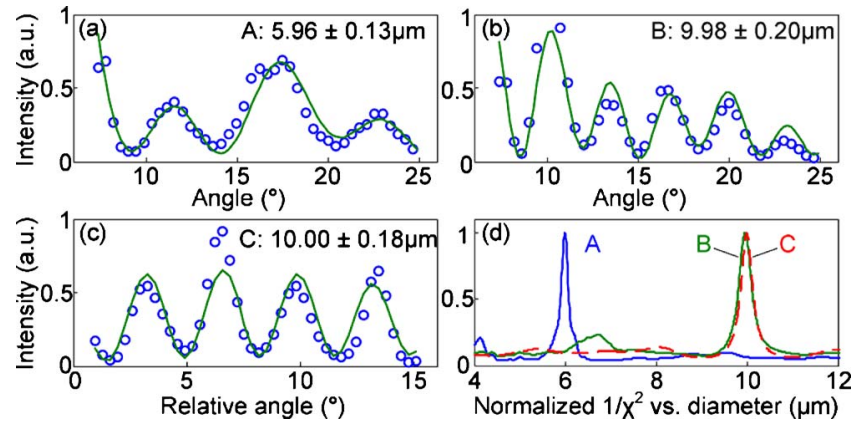

Fig. 3. (Color online) Mie fitting results along lines (a) A, (b) B, and (c) C. (d) Normalized $1 / \chi^{2}$ values indicating best fits in diameter.

tained using other scattering directions and polarizations. These examples show that a single data acquisition by the FITS system improves fitting accuracy to a few tens of nanometers and reduces fitting errors to less than $200 \mathrm{~nm}$ as compared with previous 1D a/LCI systems [8,9], which required multiple scattering measurements to achieve the same accuracy and precision for structural measurements.

We have introduced the FITS a/LCI system for polarization-sensitive measurement of $2 \mathrm{D}$ angular scattering. The MSI configuration is simple and easy to implement. Compared with previous $1 \mathrm{D}$ a/LCI systems, the FITS system can acquire scattering with two more degrees of freedom, i.e., polarization and a second angular direction, both of which are made possible by selecting $R_{1}$ as the reference signal. Preliminary results demonstrate the system's potential as a powerful tool for advanced study of light scattering. The $2 \mathrm{D}$ data reveal fine details of the scattering patterns and offer versatility in signal analysis. Fitting at different areas of the 2D distribution is likely to provide improved knowledge about scattering samples and may be particularly useful for analysis of aspherical scatterers.

This work is supported by the National Institutes of Health (NIH) (National Cancer Institute) under grants R33-CA 109907 and R01CA138594.

\section{References}

1. V. Backman, V. Gopal, M. Kalashnikov, K. Badizadegan, R. Gurjar, A. Wax, I. Georgakoudi, M. Mueller, C. W. Boone, R. R. Dasari, and M. S. Feld, IEEE J. Sel. Top. Quantum Electron. 7, 11252 (2001).

2. C. S. Mulvey, A. L. Curtis, S. K. Singh, and I. J. Bigio, IEEE J. Sel. Top. Quantum Electron. 13, 1663 (2007).

3. D. Arifler, I. Pavlova, A. Gillenwater, and R. RichardsKortum, Biophys. J. 92, 3260 (2007).

4. J. D. Wilson and T. H. Foster, Opt. Lett. 30, 2442 (2005).

5. Z. J. Smith and A. J. Berger, Opt. Lett. 33, 714 (2008).

6. W. J. Brown, J. W. Pyhtila, N. G. Terry, K. J. Chalut, T. A. D'Amico, T. A. Sporn, J. V. Obando, and A. Wax, IEEE J. Sel. Top. Quantum Electron. 14, 88 (2008).

7. Y. Zhu, N. G. Terry, J. T. Woosley, N. J. Shaheen, and A. Wax, J. Biomed. Opt. (to be published).

8. Y. Zhu, N. G. Terry, and A. Wax, Opt. Lett. 34, 3196 (2009).

9. J. W. Pyhtila and A. Wax, Appl. Opt. 46, 1735 (2007). 